\title{
Strategies for Effectively Visualizing 3D Flow with Volume LIC
}

\author{
Victoria Interrante* and Chester Grosch ${ }^{\dagger}$ \\ *Institute for Computer Applications in Science and Engineering \\ ${ }^{\dagger}$ Old Dominion University
}

\begin{abstract}
This paper discusses strategies for effectively portraying 3D flow using volume line integral convolution. Issues include defining an appropriate input texture, clarifying the distinct identities and relative depths of the advected texture elements, and selectively highlighting regions of interest in both the input and output volumes. Apart from offering insights into the greater potential of 3D LIC as a method for effectively representing flow in a volume, a principal contribution of this work is the suggestion of a technique for generating and rendering 3D visibilityimpeding "halos" that can help to intuitively indicate the presence of depth discontinuities between contiguous elements in a projection and thereby clarify the $3 \mathrm{D}$ spatial organization of elements in the flow. The proposed techniques are applied to the visualization of a hot, supersonic, laminar jet exiting into a colder, subsonic coflow.
\end{abstract}

\section{INTRODUCTION}

Line Integral Convolution (LIC) [4] offers an elegant means of effectively visualizing directional information. Although most frequently used to represent 2D flow, or flow across a surface in 3D [8, 3, 22, 14, 11], LIC methods can equivalently be employed to illustrate flow in a volume $[4,18]$. The popularity of LIC as a tool for 3D flow visualization has historically been limited, however, by the difficulties inherent in clearly and effectively portraying dense volume textures. This paper discusses some of the factors that may underlie the perceptual difficulties that we often encounter in understanding dense 3D displays and describes several techniques that can be used to enhance the clarity of volume-rendered LIC images of 3D flow. Although this paper is most directly concerned with the representation of 3D flow via LIC, many of the issues and methods that we discuss are relevant to the representation of $3 \mathrm{D}$ flow by other related means, such as densely clustered geometric streamlines, or hedgehog icons.

\section{BACKGROUND AND MOTIVATION}

LIC methods define the value at each point in an output texture image as a weighted sum of the values that lie at evenly spaced intervals along the streamline originating at the corresponding point in an input texture image and extending in both directions. Stalling and Hege [20] describe a particularly clear and efficient algorithm for computing LIC, and theirs is the general approach (extended to 3D) that we follow in this work.

When applied to 3D data, LIC methods offer the potential to generate a volumetric texture that provides a full, immediate and intuitive impression of the global and local character of a 3D flow. Volume LIC can be a useful visualization option when little $a$ priori information about specific flow features is available, when it is not easy or practical to define a single surface or set of surfaces that adequately captures all of the relevant features of a
3D flow, when it is feared that critical flow features may be overlooked in an interactive inspection of the data via selectively chosen streamlines, or when one needs to produce a single printed image that portrays, as completely and elegantly as possible, the aggregate contents of a 3D flow dataset. Volume LIC is, of course, but one of many viable options available for visualizing 3D flow; in particular, several other volumetric texturing techniques have been previously proposed by Crawfis, Max and colleagues $[5,6,15]$.

\section{DEFINING THE INPUT TEXTURE}

In 2D implementations, LIC is typically used to locally correlate the color values of an input texture in the direction of the flow over a surface, and a standard input in this case would be an opaque texture of uncorrelated white noise. For maximum effectiveness in 3D applications, however, it is beneficial to begin with a sparsely opaque input texture, and to use LIC to correlate both color and opacity values in the direction of the flow through the volume. When the input texture is defined as a sparse set of evenly-distributed points [25], one can effectively use LIC to scan-convert a set of evenly-distributed streamlines. By advecting the "empty" space along with the full, it is possible to minimize problems with clumping, and to finesse to some extent, the very difficult problem [24] of determining appropriate streamline seedpoints and lengths for a pleasingly even coverage of the $3 \mathrm{D}$ space. Best results can be achieved if the input spots are randomly situated in the volume according to an approximate Poisson-disk distribution, rather than laid out on a uniform grid or distributed by a purely random or jittered sampling method [11]. In some instances it can also be desirable to confine the distribution of input texture elements to specific, predefined regions of interest.

\section{PRELIMINARY RESULTS}

Fig. 1 shows a preliminary application of volume LIC to the visualization of a 3D flow. The depicted data represents a numerical simulation [10] of a hot, supersonic, laminar jet (temperature $=490 \mathrm{~K}$, Mach number $=1.57$ ) exiting into a colder, subsonic coflow (temperature $=300 \mathrm{~K}$, Mach number $=0.63$ ); the objective of the simulation is to investigate the potential effectiveness of using tabs in jets to reduce engine noise.

To concentrate the highest texture opacities in regions of fastest flow and to reduce to values approaching zero the representation of the texture where the flow was relatively slowest, we premultiplied the input texture to the volume LIC by a function of the magnitude of the velocity of the flow. Note that this is not the same thing as showing the flow over an isosurface of velocity magnitude; each streamline is represented in its entirety and in its true spatial orientation, and interior as well as exterior regions of the flow are portrayed. 


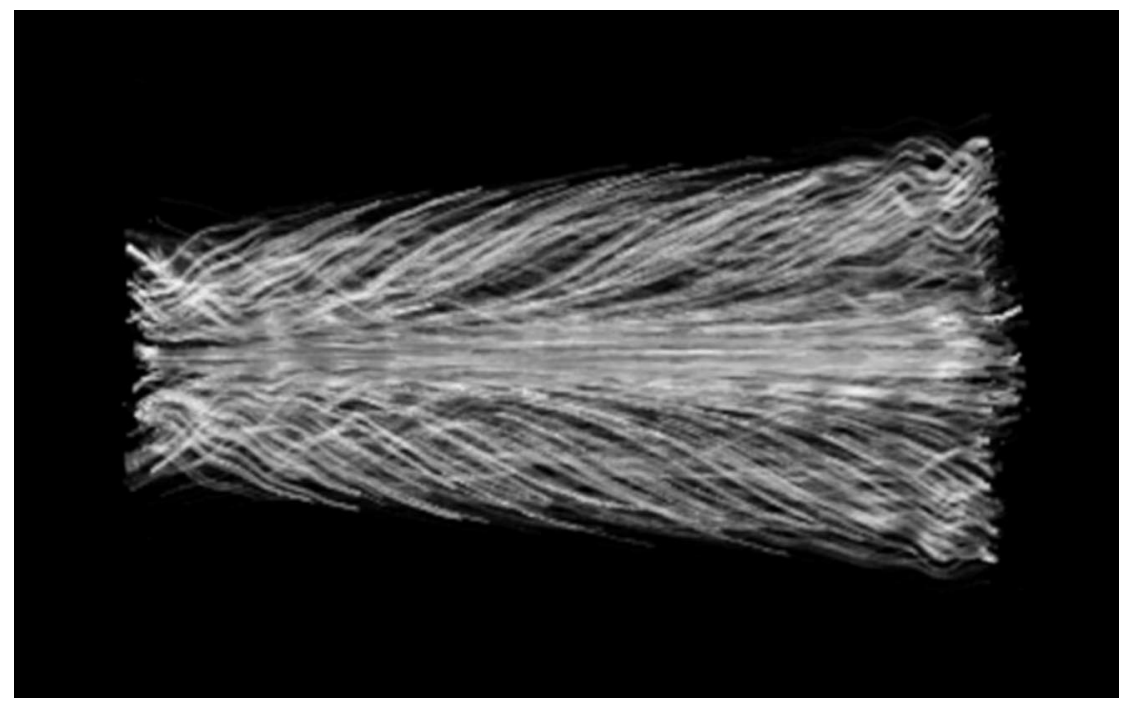

Figure 1: A volume-rendered image of a 3D texture generated by volume LIC from an input texture of evenlydistributed random point samples, premultiplied by a function of the velocity magnitude. Shading is computed as a function of the angle between the flow direction and the light direction, as motivated by [27], [2], and others. Note, nevertheless, the lack of a clear indication of the local depth order relations between overlapping and adjacent lines.

\section{CLARIFYING THE DISPLAY}

The goal of volume LIC is to effectively represent a 3D flow via a dense 3D texture. However, it can often be extremely difficult to adequately perceive the spatial organization of the discrete elements in such an image.

It has long been recognized that, in the absence of indications to the contrary, objects will generally appear to lie on the background over which they are superimposed [9]; a prime example of this phenomenon is the immediate sense of flattening that occurs when the motion of a set of dots or other tiny unshaded elements representing a $3 \mathrm{D}$ object is halted [16]. In the absence of motion and/or stereo cues, it becomes particularly important to take as full advantage as possible of pictorial techniques such as shading and shadows for conveying information about the relative positions and orientations of the elements in a dense display. Zöckler, Stalling and Hege [27, 21] demonstrate a particularly appropriate technique for determining the shading of a streamline, and this is the general illumination model (also described by Banks [2] and others [1]) that we have adopted. Even so, it can be difficult to accurately and efficiently interpret the spatial relationships between similarly oriented adjacent or overlapping lines. Although cast shadows can be useful for explicitly specifying the depth distance of an object superimposed over a horizontal groundplane [26], their effectiveness for indicating the relative depths of the various elements in a dense volume texture is somewhat limited by the difficulty of reliably determining the correspondence between any individual element and the shadows it generates on its neighbors.

In the subsections that follow, we discuss several techniques that have proven useful for clarifying the display of volume textures generated via 3D LIC.

\subsection{Differentiating the Distinct Elements}

One of the most important concerns in clarifying a dense display is the need to convey the distinct identities of adjacent or overlapping elements. Streamline shading according to the Phong illumination model described by $[27,2$, et. al] can be used to clearly depict the local orientation of a flow, but cannot effectively differentiate streamlines that are separated in depth but which flow in a similar direction. Unless the individual lines are assigned different material colors, or are allowed to randomly vary in luminance (as is the case when the seedpoints are randomly distributed and the opacity of a streamline is allowed to fall off with distance from the origin), similarly oriented, densely clustered lines will be largely indistinguishable from each other in a projected view, and will appear to coalesce into a unified solid surface of indeterminate depth. Standard depth cueing techniques can be somewhat helpful for differentiating very near from very far elements, but are less useful for indicating the kinds of subtle depth differences that more often predominate in these densely populated displays.

By assigning each of the different input texture elements one of a small number of harmonious yet readily distinguishable different hues, as demonstrated in color plate 1 , it may also be possible to facilitate the focusing of selective attention on different subsets of the LIC-generated lines in turn. Colors can be randomly distributed among the input points, as shown here, or may alternatively be assigned on the basis of a function computed either over the streamline or at its point of origin. When the colors are systematically assigned, random luminance variations need to be introduced to prevent the perceptual clumping of elements in homogeneously-valued regions.

\subsection{Emphasizing the Depth Discontinuities}

Even when the elements of a volume texture are appropriately shaded, and can be readily differentiated from each other on the basis of color and/or luminance variations, it can still be difficult to accurately and intuitively appreciate the existence and the magnitude of the depth separation between nearby elements in a projected view. An important technique for improving the clarity of the display in such cases is to explicitly emphasize the existence of the depth discontinuities [17]. 

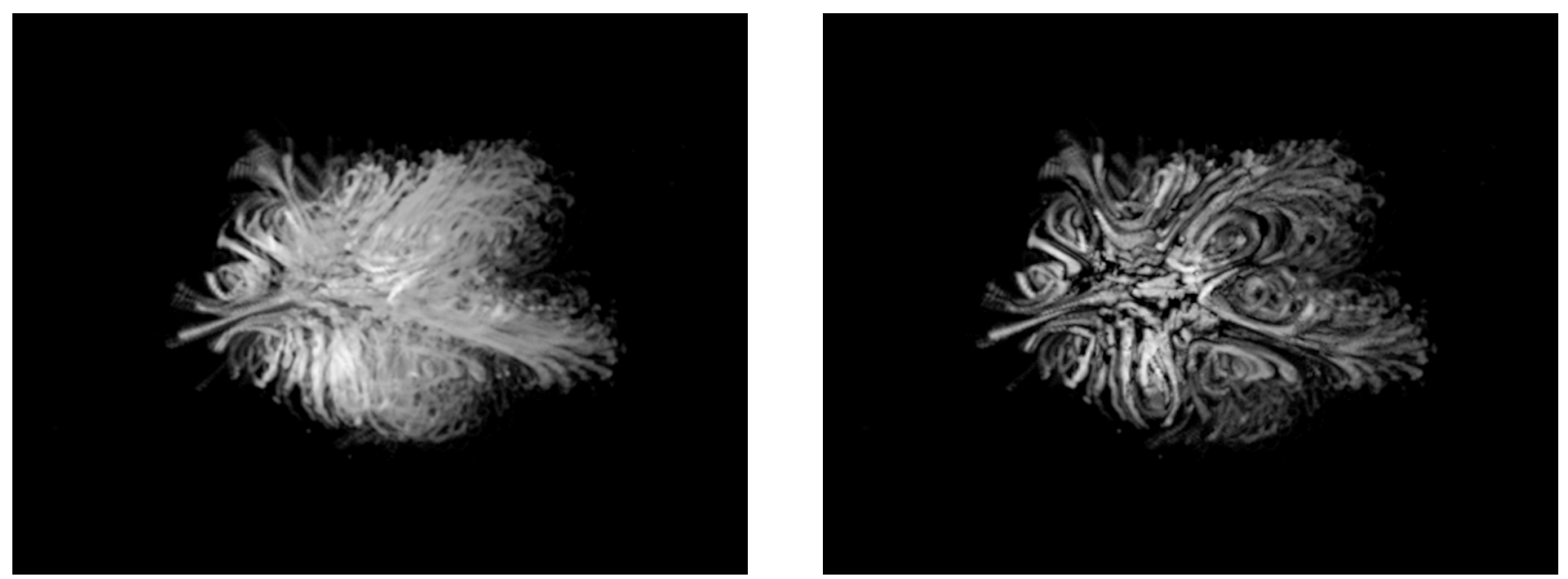

Figure 2: An oblique view of the flow, rendered with (right) and without (left) visibility-impeding 3D halos.

In ordinary binocular vision, depth discontinuities are signaled by the presence of "unpaired" regions in the views from each eye [19]. Artists and illustrators have long exploited this correspondence by using gaps to indicate situations in which one line crosses behind another, and Dooley and Cohen [7], among others, have suggested the importance of incorporating such a technique into computer-generated line drawing representations.

Because we are using volume rendering for this application, and the presence of an "occluding element" will be indicated by a continuous rather than a binary function, it is most appropriate to use a similarly continuous metric to define the locations and strengths of the gaps that will mark the depth discontinuities. The approach that we use is to define a visibility-impeding $3 \mathrm{D}$ volumetric "halo function" via what can be characterized as a variation on the theme of multi-frequency LIC [12]: a second input texture, identical to the first except that it contains slightly larger scan-converted spots at the same locations as the smaller spots in the original input, is also integrated in the direction of the flow. Since the flow is identical over the two textures, the streamlines need be computed just once, and only the indexing and summation steps have to be repeated. The second output texture, which represents the volume occupied by the first output texture, plus a smoothly-defined, scan-converted margin, can then be used during volume rendering to decrease the contribution to the final image of any voxel encountered after a halo has been entered and subsequently exited. (Because every line will be surrounded by its own halo, it is important to allow the voxels lying between the entrance and exit points of the first-encountered halo to be rendered in the normal fashion.) We impede the visibility of voxels encountered after the first halo exit by increasing the value of the accumulated opacity along the ray by an amount proportional to the strengths of the halos that have been passed, calculated as the maximum of the halo values encountered so far along this ray. It should be noted that this implementation will fail to indicate the existence of depth discontinuities between lines whose halos overlap in 3-space, even if the lines themselves do not actually intersect. Fig. 2, and color plate 1 , illustrate the extent to which volume halos can clarify the spatial relationships between inexactly-defined overlapping lines in a fairly low resolution $(202 \times 202 \times 290)$ dataset, without introducing inappropriately hard edges or jaggies.

\subsection{Facilitating Local Depth Order Judgments}

Recent research [13, 23] indicates that our understanding of surface shape may be derived not from an aggregation of individual estimates of the surface normal directions at distributed points, but from the organization of space defined by local depth order relationships between adjoining regions. It follows that the perception of the relative positions and orientations of the individual elements in a single, monocular view of a dense 3D texture would be particularly difficult when there is a lack of continuity information in the projected scene. The depth of any individual element in a dense 3D texture cannot be inferred from the depths of other elements in its general vicinity, as can be done when short line segments or points are arranged across a rigid surface, and in the absence of supplementary information it is difficult to infer the 3D orientation of a randomly positioned line segment solely from its projected length and orientation in an individual perspective view. The problem of generating a comprehensible still image of a 3D flow volume is substantially exacerbated, then, when the texture is comprised of small points or short tufts, as shown in fig. 3 and color plate 2. Adding kinetic depth cues, for example via object rotation, is of less assistance for clarifying the display when the spatial correspondence between the discrete elements is so unclear. The better solution is to use stereo, or to animate the tufts so that they follow the flow, and thereby provide the needed continuity information.

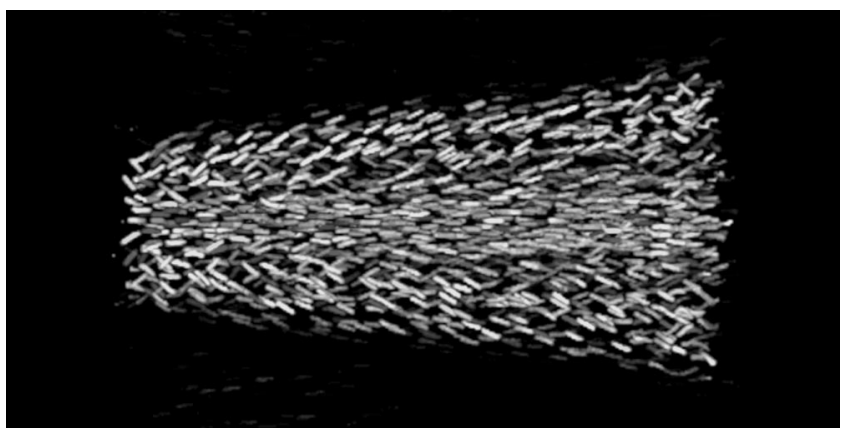

Figure 3: Local depth order relations are particularly difficult to appreciate when continuity information is lacking, even if halos and color variations are used. 


\subsection{Conveying Multiple Variables over the Flow}

When streamlines are made available as a scan-converted volume texture, one has the option of easily employing standard volume rendering procedures to isolate specific regions of interest or to convey information about related scalar quantities over the flow, as shown in color plate 3 , by modifying the local color and/or opacity values at any given point in the volume according to functions defined over registered datasets.

Adopting such an approach can facilitate the exploration of a wide variety of display options using volume LIC. It's important to stress that computing the full 3D LIC can be a fairly timeconsuming process, sometimes taking up to an hour or more, depending on the desired resolution of the output texture. Fortunately, rendering a precomputed flow texture using different mappings of colors and opacities can be done relatively quickly, though perhaps not necessarily at interactive rates.

\section{CONCLUSIONS}

Volume LIC offers a viable option for visualizing 3D flow. With such an approach, one has the potential to automatically capture many of the global and local features of a flow while largely sidestepping the potentially difficult problem of defining an appropriate distribution of streamlines through the volume. Once a scan-converted texture has been computed, a variety of different color and/or opacity mappings can be used to incorporate additional information about related flow parameters.

The comprehensibility of the dense 3D textures produced by volume LIC can be significantly improved when longer streamlines are used to represent the flow rather than shorter tufts, whose local depth order relationships can be particularly difficult to discriminate; by using random or evenly-distributed color and/or luminance variations in addition to flow-based shading to differentiate the distinct identities of potentially adjacent or overlapping texture elements while providing cues to their spatial orientation; and by explicitly signaling the presence of depth discontinuities between overlapping elements in a projected view via the use of smoothly-defined, visibility-impeding 3D halos.

\section{ACKNOWLEDGMENTS}

This work was supported by ICASE under NASA contract NAS1-19480. We are grateful to Marc Levoy for providing the original volume rendering platform upon which this work was implemented, and to Kwan-Liu Ma, Hans-Christian Hege, David Banks, and the anonymous reviewers for helpful discussions and suggestions.

\section{REFERENCES}

[1] Ken-ichi Anjyo, Yoshiaki Usami and Tsuneya Kurihara. "A Simple Method for Extracting the Natural Beauty of Hair", Computer Graphics, vol. 26, no. 2 (Proceedings of SIGGRAPH 92), pp. 111120.

[2] David C. Banks. "Illumination in Diverse Codimensions", Computer Graphics Proceedings, Annual Conference Series, 1994, pp. 327-334.

[3] Henrik Battke, Detlev Stalling and Hans-Christian Hege. "Fast Line Integral Convolution for Arbitrary Surfaces in 3D", Visualization in Mathematics, H.-C. Hege and K. Polthier, eds., Springer-Verlag, 1997.
[4] Brian Cabral and Leith (Casey) Leedom. "Imaging Vector Fields Using Line Integral Convolution", Computer Graphics Proceedings, Annual Conference Series, 1993, pp. 263-269.

[5] Roger Crawfis and Nelson Max. "Direct Volume Visualization of Three-Dimensional Vector Fields", 1992 Workshop on Volume Visualization, pp. 55-60.

[6] Roger Crawfis and Nelson Max. "Texture Splats for 3D Scalar and Vector Field Visualization”, proc. Visualization '93, pp. 261-266.

[7] Debra Dooley and Michael F. Cohen. "Automatic Illustration of 3D Geometric Models: Lines", Computer Graphics, vol. 24, no. 2, (1990 Symposium on Interactive 3D Graphics) March 1990, pp. 77-82.

[8] Lisa K. Forsell. "Visualizing Flow Over Curvilinear Grid Surfaces Using Line Integral Convolution”, proc. Visualization '94, pp. 240247.

[9] James J. Gibson. The Perception of the Visual World, Houghton Mifflin, 1950.

[10] Chester E. Grosch, J. M. Seiner, M. Y. Hussaini and T. L. Jackson. "Numerical Simulation of Mixing Enhancement in a Hot Supersonic Jet", Physics of Fluids, vol. 9, no. 4, 1997, pp. 1125-1143.

[11] Victoria Interrante. "Illustrating Surface Shape in Volume Data via Principal Direction-Driven 3D Line Integral Convolution", to appear in Computer Graphics Proceedings, Annual Conference Series, 1997.

[12] Ming-Hoe Kiu and David C. Banks. "Multi-Frequency Noise for LIC", proc. Visualization '96, pp. 121-126.

[13] Jan J. Koenderink and Andrea J. van Doorn. "Relief: pictorial and otherwise", Image and Vision Computing, vol. 13, no. 5, June 1995, pp. 321-334.

[14] Xiaoyang Mao, Makoto Kikukawa, Noboru Fujita and Atsumi Imamiya. "Line Integral Convolution for Arbitrary 3D Surfaces Through Solid Texturing", proc. Eighth Eurographics Workshop on Visualization in Scientific Computing, Boulogne sur Mer, France, April 28-30, 1997.

[15] Nelson Max, Roger Crawfis and Charles Grant. "Visualizing 3D Velocity Fields Near Contour Surfaces", proc. Visualization '94, pp. 248-255.

[16] Brian Rogers and Maureen Graham. "Motion Parallax as an Independent Cue for Depth Perception", Perception, vol. 8, 1979, pp. $125-134$

[17] Takafumi Saito and Tokiichiro Takahashi. "Comprehensible Rendering of 3-D Shapes", Computer Graphics, vol. 24, no. 4. (proc. SIGGRAPH '90), pp. 197-206.

[18] Han-Wei Shen, Christopher R. Johnson and Kwan-Liu Ma. "Visualizing Vector Fields Using Line Integral Convolution and Dye Advection", 1996 Symposium on Volume Visualization, pp. 63-70.

[19] Shinsuke Shimojo and Ken Nakayama. "Real World Occlusion Constraints and Binocular Rivalry", Vision Research, vol. 30, no. 1, 1990, pp. 69-80.

[20] Detlev Stalling and Hans-Christian Hege. "Fast and Resolution Independent Line Integral Convolution", Computer Graphics Proceedings, Annual Conference Series, 1995, pp. 249-256.

[21] Detlev Stalling, Malte Zöckler and Hans-Christian Hege. "Fast Display of Illuminated Field Lines", IEEE Transactions on Visualization and Computer Graphics, vol. 3, no. 2, April-June, 1997, pp. 118-128.

[22] Christian Teitzel, Robert Grosso and Thomas Ertl. "Line Integral Convolution on Triangulated Surfaces", proc. Fifth International Conference in Central Europe on Computer Graphics and Visualization (WSCG '97), Plzen-Bory, Czech Republic, Feb. 10-14, 1997, pp. 572-581.

[23] James T. Todd and Francene D. Reichel. "Ordinal Structure in the Visual Perception and Cognition of Smoothly Curved Surfaces", Psychological Review, vol. 96, no. 4, 1989, pp. 643-657.

[24] Greg Turk and David Banks. "Image-Guided Streamline Placement", Computer Graphics Proceedings, Annual Conference Series, 1996, pp. 453-460.

[25] Rainer Wegenkittl, Eduard Gröller and Werner Purgathofer. "Animating Flowfields: Rendering of Oriented Line Integral Convolution", to appear in proc. Computer Animation '97, Geneva, Switzerland, June 5-6, 1997.

[26] Albert Yonas, Lynn T. Goldsmith and Janet L. Hallstrom. "Development of Sensitivity to Information Provided by Cast Shadows in Pictures", Perception, vol. 7, no. 3, 1978, pp. 333-341.

[27] Malte Zöckler, Detlev Stalling and Hans-Christian Hege. "Interactive Visualization of 3D-Vector Fields Using Illuminated Streamlines", proc. Visualization '96, pp. 107-113. 


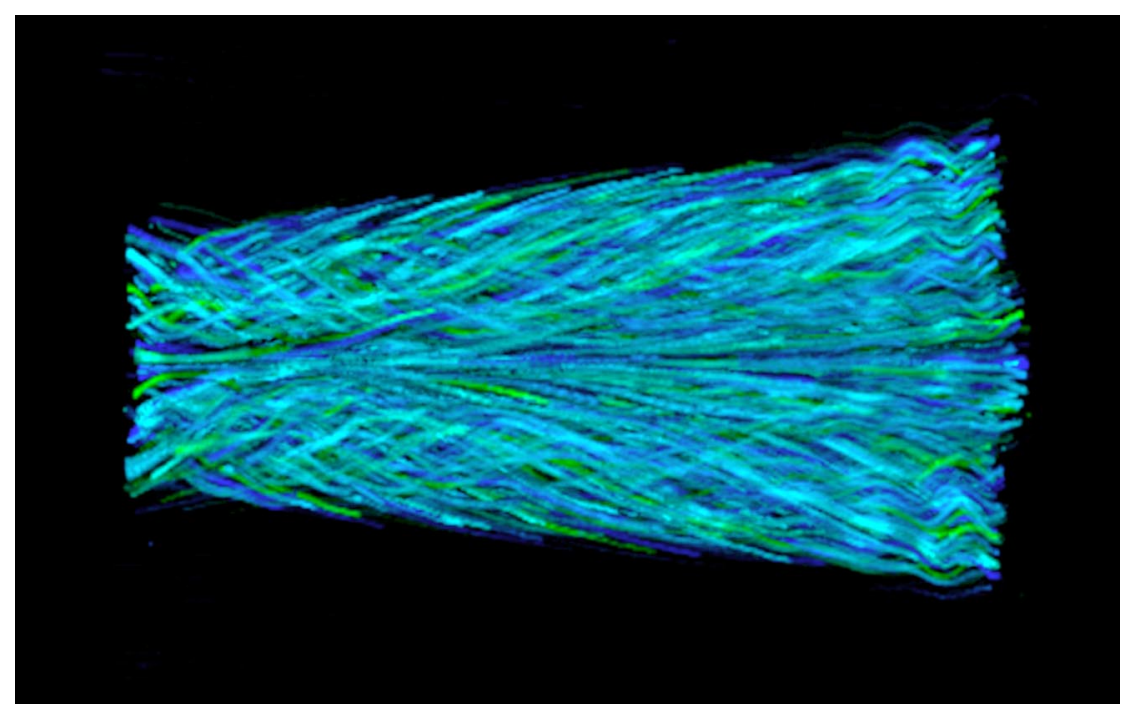

Color Plate 1: A volume rendered image of a 3D flow dataset generated using volume LIC. Color variations are used to differentiate the individual streamlines and to facilitate the conscious direction of focused attention to subsets of these lines. Depth discontinuities are explicitly indicated by means of a locally-defined 3D "halo" that impedes the visibility of underlying texture elements in the vicinity of overlying lines.

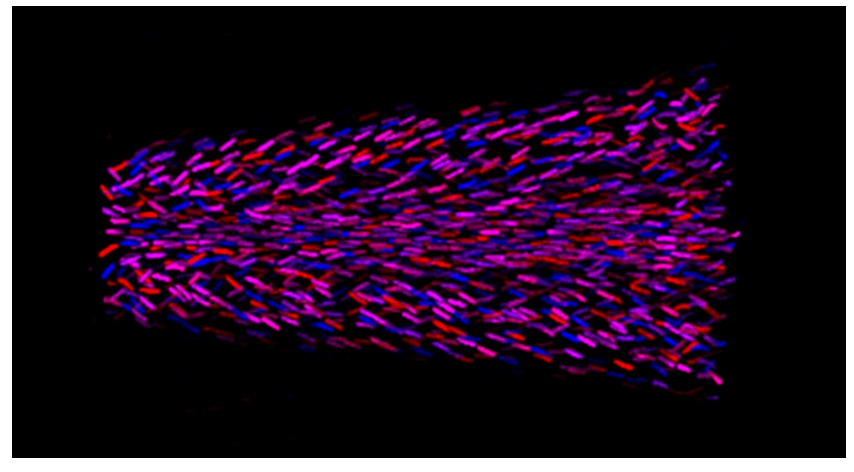

Color Plate 2: Local depth order relationships are difficult to appreciate when continuity information is lacking, even if halos and color variations are used.

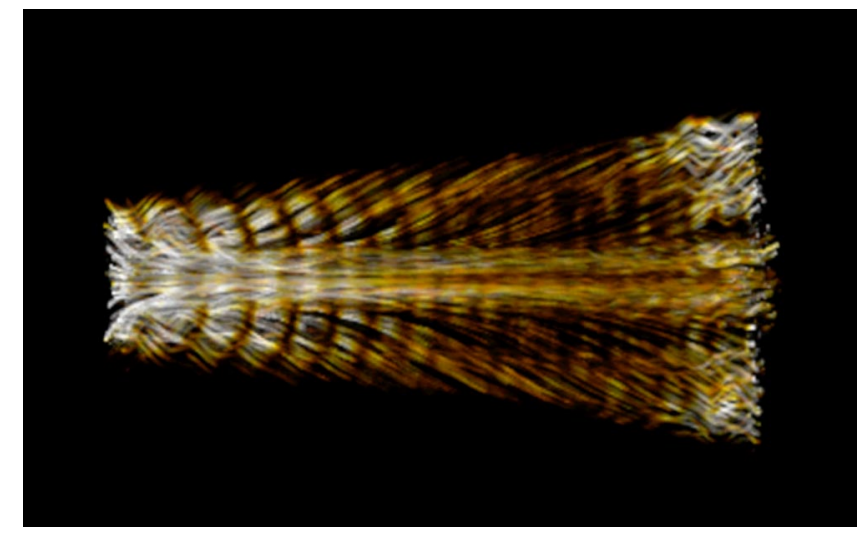

Color Plate 3: When streamlines are represented as a volume texture, color and opacity information can be easily redefined based on any function of the flow values across the volume. In this image, vorticity magnitude is locally mapped to streamline color using a heated-object colorscale. The striations along the axial direction reveal the presence of periodic waves propagated down the jet axis. 